\title{
Clinical study of patients with arthropathy due to craniomandibular disorders
}

\author{
Mariana Dimova-Gabrovska ${ }^{1}$, Desislava Dimitrova ${ }^{2}$, \\ Bozhidar Yordanov ${ }^{1}$, Todor Uzunov ${ }^{1}$, Nikolay Apostolov ${ }^{1}$
}

1. Department of Prosthetic Dentistry, Faculty of Dental Medicine, Medical University Sofia;

2. Private practice - Sofia, Bulgaria;

\begin{abstract}
Craniomandibular disorders affect the structures of the masticatory system and manifest themselves with variations in the volume of movement, changes in strength characteristics, pain symptoms, and more.
\end{abstract}

Purpose of this study is to examine the changes in the masticatory system of patients suffering from arthropathy due to craniomandibular dysfunction, through clinical functional analysis.

Material and Methods: 30 patients with signs of arthropathy and 30 healthy patients with no signs of pathology were studied. All study participants underwent clinical functional and manual analysis using DGFDT protocols.

Results: $43,3 \%$ of the examined patients reported objective sensation of pain during lateral palpation of TMJ, 40\% - during dorsal palpation of TMJ, 50\% - during cranial compression of TMJ, 40\% - during dorsal compression of TMJ and 60\% - during medial translation.

Conclusion: Clinical functional analysis allows reliable and purposeful diagnosis in patients with arthropathy.

Keywords: functional analysis, craniomandibular disorders, arthropathy 


\section{Introduction}

Craniomandibular disorders (CMD) is a term meaning the functional pathology of the masticatory system, including clinical symptoms of the chewing muscles, temporomandibular joint (TMJ) and adjacent structures [1]. The signs and/or symptoms of CMD are widespread - between 16 and 59\% of the total population [2], which defines the topic as relevant and significant.

Diagnosis of the masticatory system `s disorders has undergone significant changes in the methods and means used [3]. Paraclinical studies such as ultrasound, electromyography, magnetic resonance imaging are excellent sources of information about the condition, function and dysfunction of the masticatory muscles and joints, but they are not applicable in daily practice [4]. The most commonly used modern methods for evaluation of the masticatory system include tests for analysis of the active and passive movements, as well as palpation of the muscles and temporomandibular joints [5]. According to the scientific literature [6], clinical functional studies have a high diagnostic value and can easily be used by dental practitioners. A number of authors have developed orthopedic tests to evaluate the function of the masticatory system [7, 8, 9]. Samples include analysis of passive movements, movements of the mandibular joints through traction and translation, as well as dynamic and static pain tests. The results obtained after loading of TMJ in a relaxed muscles condition give information about the functioning of the joint complex, static tests serve to evaluate the condition of the chewing muscles, and the dynamic pain tests provide information about the activity of the whole masticatory system (joints and muscles).

Over the last decade, medical science has demonstrated the existence of interrelationships that define craniomandibular disorders not only as dysfunctional conditions and syndromes affecting the maxillofacial region, but as dysfunctional diseases with convergences in the musculoskeletal, vestibular and hormonal system, which affect the homeostasis of the whole body, both in the waking state and during sleep [10, 11, 12]. All these justify the need for easy, rapid and focused early diagnosis, which creates objective prerequisites for early recognition of the functional pathology and treatment.

\section{Aim}

The purpose of this study is to examine the changes in the masticatory system of patients suffering from arthropathy due to craniomandibular disorders, through clinical functional analysis.

\section{Material and Methods}

After the initial examination of the basic structures of the chewing apparatus among 200 patients (between 35 and 55 years of age) 2 groups were selected - control group and experimental group:

- $\quad$ the experimental group include 30 patients (12 males and 18 females) with a mean age of $43 \pm 4.7$ years, with signs and/or symptoms of arthropathy reported;

- $\quad$ the control group included 30 healthy patients (14 men and 16 women) with a mean age of $41 \pm 3.5$ years with normal occlusion according to Andrews, with no signs and symptoms of craniomandibular disorders. 
All participants were examined by clinical functional and manual analysis using the DGFDT protocols [13, 14].

A comparative assessment was made between patients without signs and symptoms of CMD and patients with established arthropathy with the following findings:

- $\quad$ the presence of noise in the TMJ;

- pain during lateral palpation of TMJ;

- $\quad$ pain during dorsal palpation of TMJ;

- $\quad$ pain during dorsal compression of the TMJ;

- pain during cranial compression of the TMJ;

- $\quad$ pain during the medial translation of the TMJ.

The obtained data were statistically processed with the package of applications for analysis of data from epidemiological and clinical studies - SPSS for Windows, version 22.0.0., as applied: descriptive analysis, variation analysis, correlation analysis and search for statistical dependence of qualitative variables by the X2-criterion (chi-square) and Fisher's exact (two-tail) test). MS Excel 2010 was used to graphically represent the results.

\section{Results}

Considering the criterion of "noise in the TMJ", a statistically significant difference was found between the experimental and control groups $(X 2=8.19, P=0.004)$. The relative share of patients with arthropathy who have noise in the TMJ is more than 7 times higher than this in the control group $(50.0 \%$ to $6.7 \%$, respectively). The likelihood of occurrence of noise in TMJ in patients with arthropathy is 7.72 times higher (RR = $7.72(1,116-53,469) ; P<0.001)$ compared to healthy subjects.

Similar results are reported with regard to the objective feeling of pain during palpation of the lateral pole of the joint head. The relative share of patients with pain in the experimental group was significantly higher than the one in the control group, $43.3 \%$ to $6.7 \%$, respectively $(X 2=6.27, P=0.012)$. The chance of pain occurrence in lateral palpation in patients with arthropathy was 6.32 times higher $(R R=6.32(0.919-43.476)$; $P<0.001)$ compared to healthy patients.

Unlike the criteria considered so far, the analysis of the results obtained from the dorsal palpation of the TMJ showed no statistically significant difference between the two groups $(x 2=1.80, p=0.157)$. However, in patients with arthropathy, the relative share of those with pain was higher than the one in healthy patients (40.0\% for the experimental group and $20.0 \%$ for the control group, respectively).

Similar results were observed with respect to the functional tests of cranial $(x 2=2.23, P=0.120)$ and dorsal $(x 2=0.189, P=0.461)$ compression. The relative share of patients with pain in the experimental group was greater than the one in the control group, both in terms of cranial compression $(50.0 \%$ vs. $26.7 \%$, respectively) and in dorsal compression (40.0\% to $33.3 \%$, respectively). 
On the other hand, the relative share of patients with arthropathy and pain in the medial translation was significantly greater $(60.0 \%)$ than in healthy patients $(33.30 \%)(X 2=2.85, P=0.045)$. The chance of discomfort occurrence in medial translation in the subjects in the experimental group was 2.09 times higher than in the control group $(\mathrm{RR}=2.09(0.850-5.145)$; $\mathrm{P}<0.05)$ (Fig. 1)

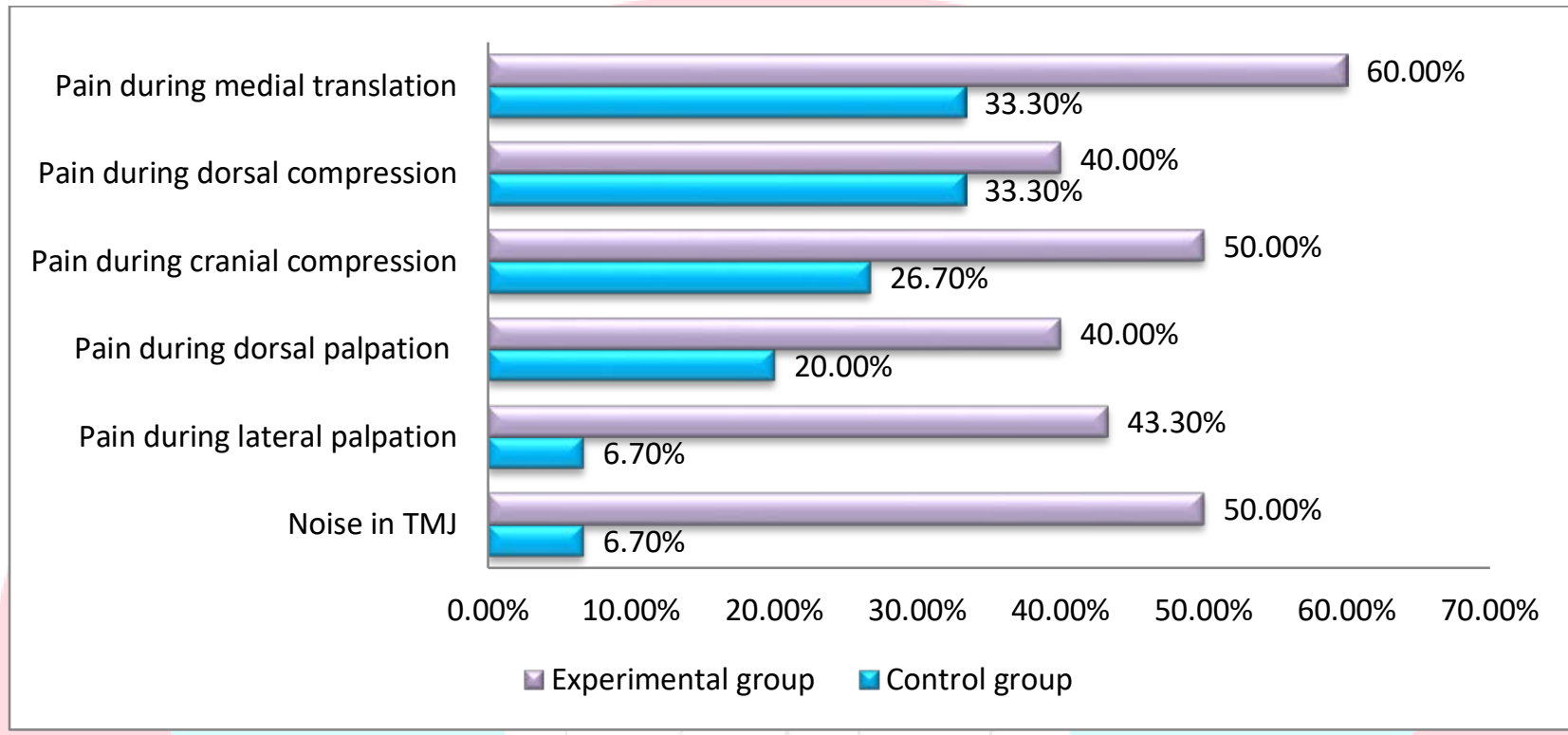

Fig. 1. Relative share of patients in the experimental and control groups with an objective sensation of pain

\section{Discussion}

The results obtained indicate that a significant proportion of patients affected by arthropathy due to CMD respond with an objective sensation of pain in palpation, compression and translation of the TMJ. Early diagnosis and recognition of the signs and symptoms of functional pathology is crucial, as it creates an objective prerequisite for the regulation of abnormalities and the restoration of physiological norm. Timely treatment ensures the functional health of the masticatory system, which prevents the chronicity of the processes with complications in other organs and systems. The applied tests of clinical functional analysis are non-invasive, rapid and easily applicable in the daily practice of the dentists. The findings are a source of reliable information on the condition of the masticatory system and allow the identification of pathological findings within a single visit without the need for specialized equipment or technique.

\section{Conclusion}

Clinical functional analysis allows reliable and purposeful diagnosis in patients with arthropathy. The results indicate a significantly more widespread and more expressed pain symptomatology in patients with arthropathy due to CMD than in healthy patients. This necessitates the inclusion of functional analysis of the masticatory system as part of a routine dental examination. 
Acknowledgments: Current data are part of the development of scientific project № 93, funded by the Medical University of Sofia, Bulgaria.

\section{References}

1. Köneke, Ch. Craniomandibuläre Dysfunktion. Interdisziplinäre Diagnostik und Therapie. Quintessenz Verlag, Berlin, 2010.

2. Bevilaqua-Grossi D, Chaves TC, de Oliveira AS, Monteiro-Pedro V. Anamnestic index severity and signs and symptoms of TMD. Cranio. 2006 Apr; 24(2):112-8.

3. Conti P, Neanes dos Santos C, Lauris J. Interexaminer agreement for miscle palpation procedures: the efficacy of calibration program. Cranio. 2002; 20(4):289-294.

4. Widmer C, Lund J, Feine J. Evaluation of diagnostic tests for CMD. CA Dent Assoc. 1990; 123: 47-54.

5. Dworkin S, Huggins K, LeReshe L, Von Korff M. Assessing clinical signs of temporomandibular disorders: Reliability of clinical examiners. J Prosthet Dent. 1990; 63(5): 574-579

6. Lobbezoo-Scholte A, Wijer A, Steenks M. Interexaminer reliability of six orthopaedic test in diagnostic subgrups of craniomandibular disorders. J Oral rehabil. 1994; 21(): 273-285.

7. Solberg W. temporomandibular disorders: Physical tests in diagnosis. Br Dent J. 1986; 160:273-277.

8. Naeije M, Hansson T. Electromyographic screening of myogenous and arthrogenous TMJ dysfunction patients. J Oral Rehabil. 1986; 13(5): 433-441

9. Steenks $M$, de Wijer $A$. Kiefergelenksfehlfunktionen aus physiotherapeutisher und zahnmedizinischer Sicht. Diagnose und therapie. Quitessenz Verlags-GmbH, Berlin., 1991.

10. Witulski S, Vogl Th, Rehart St, Ottl P. Evaluation of the TMJ by means of Clinical TMD examination and MRI diagnostics in patients with rheumatoid arthritis. Biomed Res Int. 2014; 2014: 328560

11. Katzberg R. Is Ultrasonography of the Temporomandibular Joint Ready for Prime Time? Is There a "Window" of Opportunity J Oral Maxillofac Surg. 2012;70(6):1310-14.

12. Christiansen G. Computer-aided measurement of the functional joint space of the temporomandibular joint. J Craniomand Func. 2014;2(4):329-43.

13. Ahlers M, Jakstat H, Freesmeyer B, Simonis A, Hugger G, Meyer G. Vorschlag eines modernen Diagnoseschemas zur therapiespezifischen Erfassung von Anamnesen und Befunden bei CMD. Online Abstract zur Jahrestagung der Arbeitsgemeinschaft für Funktionslehre 2001. DGZMK, 2001.

14. Freesmeyer W, Fussnegger M, Ahlers M. Diagnostic and therapeutic - restorative procedure for masticatory dysfunctions, GMS Current Topics in Otorhinolaryngology - Head and Neck Surgery. 2005; 4(19): 1-29.

\section{Corresponding author:}

\section{Desislava Dimitrova,}

Private practice,

Hrizantema 20 str, Sofia, Bulgaria

tel: +359 887517892

email: desislavvva23@gmail.com 